\title{
Discrete kink dynamics in hydrogen-bonded chains: The two-component model
}

\author{
Karpan, V.M.; Zolotaryuk, Yaroslav; Christiansen, Peter Leth; Zolotaryuk, Alexander
}

Published in:

Physical Review E

Link to article, DOI:

10.1103/PhysRevE.70.056602

Publication date:

2004

Document Version

Publisher's PDF, also known as Version of record

Link back to DTU Orbit

Citation (APA):

Karpan, V. M., Zolotaryuk, Y., Christiansen, P. L., \& Zolotaryuk, A. (2004). Discrete kink dynamics in hydrogenbonded chains: The two-component model. Physical Review E, 70(5), 056602.

https://doi.org/10.1103/PhysRevE.70.056602

\section{General rights}

Copyright and moral rights for the publications made accessible in the public portal are retained by the authors and/or other copyright owners and it is a condition of accessing publications that users recognise and abide by the legal requirements associated with these rights.

- Users may download and print one copy of any publication from the public portal for the purpose of private study or research.

- You may not further distribute the material or use it for any profit-making activity or commercial gain

- You may freely distribute the URL identifying the publication in the public portal

If you believe that this document breaches copyright please contact us providing details, and we will remove access to the work immediately and investigate your claim. 


\title{
Discrete kink dynamics in hydrogen-bonded chains: The two-component model
}

\author{
V. M. Karpan, ${ }^{1,2}$ Y. Zolotaryuk, ${ }^{1,2}$ P. L. Christiansen, ${ }^{1}$ and A. V. Zolotaryuk ${ }^{1,2}$ \\ ${ }^{1}$ Section of Mathematical Physics, IMM, Technical University of Denmark, DK-2800 Lyngby, Denmark \\ ${ }^{2}$ Bogolyubov Institute for Theoretical Physics, 03143 Kyiv, Ukraine \\ (Received 17 April 2003; revised manuscript received 6 April 2004; published 9 November 2004)
}

\begin{abstract}
We study discrete topological solitary waves (kinks and antikinks) in two nonlinear diatomic chain models that describe the collective dynamics of proton transfers in one-dimensional hydrogen-bonded networks. The essential ingredients of the models are (i) a realistic (anharmonic) ion-proton interaction in the hydrogen bond, (ii) a harmonic coupling between the protons in adjacent hydrogen bonds, and (iii) a harmonic coupling between the nearest-neighbor heavy ions (an isolated diatomic chain with the lowest acoustic band) or instead a harmonic on-site potential for the heavy ions (a diatomic chain subject to a substrate with two optical bands), both providing a bistability of the hydrogen-bonded proton. Exact two-component (kink and antikink) discrete solutions for these models are found numerically. We compare the soliton solutions and their properties in both the one- (when the heavy ions are fixed) and two-component models. The effect of stability switchings, discovered previously for a class of one-component kink-bearing models, is shown to exist in these twocomponent models as well. However, the presence of the second component, i.e., the softness of the heavy-ion sublattice, brings principal differences, like a significant difference in the stability switchings behavior for the kinks and the antikinks. Water-filled carbon nanotubes are briefly discussed as possible realistic systems, where topological discrete (anti)kink states might exist.
\end{abstract}

DOI: 10.1103/PhysRevE.70.056602

PACS number(s): 05.45.Yv, 05.45.-a, 05.60.Cd

\section{INTRODUCTION}

Hydrogen-bonded (HB) crystals or chains have been the object of many scientific investigations in physics, chemistry, and biology in the last decades. One of the important aspects of studying these systems is the proton transport that takes place through the hydrogen bonds. The proton conductivity in HB networks is remarkably high and, in fact, some of these materials have been called protonic semiconductors because the observed proton mobility is of a comparable order with the electronic mobility in some semiconductors. For extensive reviews and papers that cover the experimental situation and the theoretical background as well as the latest bibliography see, for instance, Refs. [1-6]. Quantummechanical aspects of proton transfers in quasi-onedimensional HB systems have been studied in a number of works [7-11], to mention a few. It is also believed that the chains of hydrogen bonds act as proton wires providing an effective pathway for the rapid translocations of protons from a proton donor to a proton acceptor in several biological systems such as bacteriorhodopsin and ATP synthase $[1,12]$. Very recent experimental and theoretical studies [13-17] on filling single-walled carbon nanotubes with water open new horizons in investigating a variety of challenging scientific problems at the nanoscale.

In simple terms, a HB chain can schematically be represented as a diatomic chain $\cdots X-\mathrm{H} \cdots X-\mathrm{H} \cdots X-\mathrm{H} \cdots$, where the hydrogen atom $\mathrm{H}$ (or proton $\mathrm{H}^{+}$) in each lattice unit is connected with its adjacent heavy ions or more generally hydroxyl groups $X$ (or $X^{-}$) via either a covalent (-) or a hydrogen $(\cdots)$ bond, forming a HB bridge $X-H \cdots X$. The molecular mechanism for the proton conduction along such a HB chain comprises two complementary processes: (i) the propagation of an ionic positive (or negative) defect, when an excess proton is transferred inside the bridge interchanging the role of the covalent and hydrogen bonds with the adjacent groups $X$, and (ii) the propagation of a bonding (Bjerrum) defect, according to which the additional degree of freedom allows the group $X-\mathrm{H}$ to rotate in such a way that the interbond proton transfer is possible along the HB chain (for more details see, e.g., Refs. $[1,18]$ ). In this paper, we focus on the motion of the ionic defects, where only intrabond proton transfers are involved.

Since the proton transfer in a HB chain is a cooperative process, it is believed that the translational motion of both the positive and negative ionic defects along the chain is essentially facilitated. To describe this effect qualitatively, an effective coupling between the protons in the nearestneighbor HB bridges may be introduced, resulting in a soliton (kink) model of the proton transport in HB chains [19-22]. Owing to the fact that the proton can be found in two degenerate equilibrium states within the HB bridge, it is reasonable to model the proton potential by the superposition of the two realistic (anharmonic) potentials of a standard form (with a single-well topology, like a Morse or a Lennard-Jones potential), placed tail-to-tail at such a distance which provides a double-well form of the proton potential [23-29], as illustrated by Fig. 1. In general, it is not necessary to construct the intrabond proton potential as a sum of two single-well potentials because the equations of motion for the chain can be written in terms of any potential $V(u, \rho)$ given as a function of the proton displacement $u$ from the middle of the HB bridge and the relative displacement of the ions $X^{+}$from their equilibrium positions [25,30].

The discrete kink states, their stability and mobility properties have been studied previously [31] in detail, where the heavy ions forming the intrabond proton potential from two Morse potentials were assumed to be fixed. In this limiting 

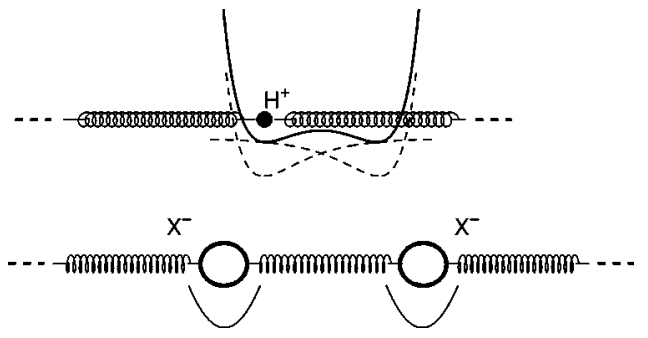

FIG. 1. Schematics of interactions in one unit cell of a $2 \mathrm{C} \mathrm{HB}$ chain.

case, which can be called the "monoatomic" limit, the ionic defects are soliton solutions to a one-component (1C) model. However, in reality, the intrabond proton potential essentially depends on the relative distance between the adjacent heavy ions that create this potential. Therefore the dynamics of such a realistic diatomic chain must be described by a twocomponent (2C) model. As a result, a number of $2 \mathrm{C}$ soliton models have been suggested, one part of which was studied in the continuum limit $[21,22,25,32-43]$, while the discreteness effects were investigated in other $2 \mathrm{C}$ models [26,28,44-53]. Even though the discreteness effects were studied for many $2 \mathrm{C}$ models used to describe the proton transport in HB chains (see, e.g., Refs. [26,51]), such a phenomenon as stability (or symmetry) switchings of the (anti)kink stationary states and related (anti)kink transmission at some velocities was not yet examined in detail for any of the 2C models. As discovered numerically by Peyrard and Remoissenet [54], the switching effect occurs due to the two factors, (i) the lattice discreteness and (ii) the shape of the on-site potential in a kink-bearing model. The typical example of this situation is the discrete nonlinear Klein-Gordon model with the Remoissenet-Peyrard substrate potential [55]. This model is one component; however, switchings of this type should occur also in a $2 \mathrm{C}$ model if the ion degrees of freedom allow the intrabond proton potential to take an appropriate shape. Even though the switching effect is expected to take place in the $2 \mathrm{C}$ case, it is not clear whether the softness of the heavy-ion sublattice does facilitate or oppose this effect. For instance, one would expect that due to selfconsistent formation of $2 \mathrm{C}$ (anti)kink states, instead of fixed switching points in the proton-proton coupling parameter, some finite intervals (windows) resulting in stronger stabilization of stability switchings could appear.

The present paper aims to proceed with the type of investigations undertaken previously [31] for the $1 \mathrm{C}$ kink model for proton transport, taking into account the degrees of freedom of the heavy-ion subsystem, which are important for the dynamics of the total two-sublattice system. Although several 2C models for proton transfers have been suggested so far, here we would like to restrict ourselves to a $2 \mathrm{C}$ model that contains a minimal number of couplings [like the conventional models such as the monoatomic or diatomic ( $\alpha$ or $\beta)$ Fermi-Pasta-Ulam chain, the $\phi^{4}$ model, or the FrenkelKontorova model], contrary to a general model studied earlier [26] by Savin and one of the authors (A.V.Z.) of this paper, where all possible nonlinearities (anharmonicities) have been involved. In the present paper we would like to consider only those (basic or characteristic) nonlinearities,

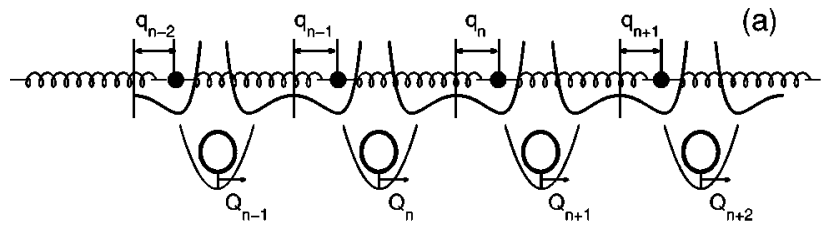

(b)

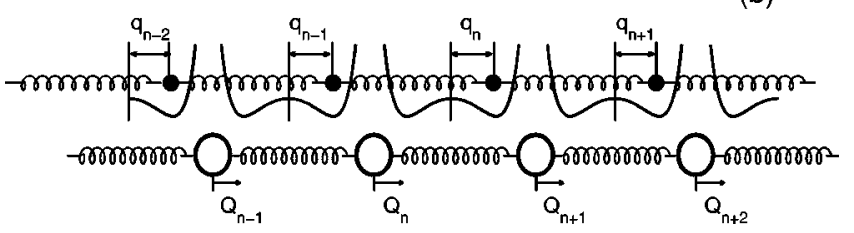

FIG. 2. Schematics of (a) $2 \mathrm{CO}$ and (b) 2CA chain models.

which are responsible for the shape formation of the proton potential. Only one anharmonicity is sufficient to describe properly a hydrogen-bonded chain, namely the ion-proton pair potential of a realistic form (e.g., a Morse or a LennardJones potential). As regards other forces, it is sufficient to consider them in an harmonic approximation. This type of modeling can be used for nanofluidic devices such as a water-filled carbon nanotube, where the on-site potential for heavy ions (oxygens) is mainly formed due to the interaction between the water molecules inside the tube and the charged carbon atoms. Another 2C model [21], where the discreteness effects have been studied in detail by Cretegny and Peyrard [51] using a collective coordinate approach, is not consistent with Fig. 1 and describes a more specific physical situation.

It is not possible to provide a double-well form of the intrabond proton potential within a standard diatomic chain, using only nearest-neighbor interactions. Therefore some additional interactions with appropriate parameters, which do not allow the adjacent heavy ions to approach very close to each other, should be involved into the model. For archetypal simplicity of the $2 \mathrm{C}$ model, it is sufficient to impose either (i) an external, single-well on-site potential for each heavy ion of the chain, periodically located at a sufficiently large distance [see Fig. 2(a)] or instead (ii) a nearest-neighbor coupling between the adjacent heavy ions, with a sufficiently strong strength and a sufficiently large equilibrium distance between them [see Fig. 2(b)]. From a physical point of view, both these cases are of interest. While the former case can describe the dynamics of a HB chain formed in a water-filled pore (e.g., in a carbon nanotube or bacteriorhodopsin), the latter case belongs mostly to quasi-one-dimensional $\mathrm{HB}$ crystals or isolated macromolecules.

Since the chain is diatomic, in both the cases, the spectrum of small-amplitude oscillations consists of two bands. In the former case, when the (decoupled) heavy ions are subject to the on-site potential, both the lower and upper bands are of the optical type, whereas in the latter case, when the coupling between the nearest-neighbor ions is included and the whole diatomic chain is totally isolated from any external environment, the lower band is of the acoustic type. Therefore we refer to these $2 \mathrm{C}$ models as the $2 \mathrm{CO}$ [see Fig. 2(a)] and 2CA [see Fig. 2(b)] models (or chains), respectively. 
The first question that arises while studying the discrete dynamics of a $2 \mathrm{C}$ HB chain is to understand how the heavyion component of the diatomic chain influences the transparency for the propagation of (anti)kinks with narrow profile. Since the proton (anti)kink component appears to be "dressed" by distortions of the heavy-ion sublattice, it is not obvious that the kink transmission effect for narrow topological solitons in the $1 \mathrm{C}$ model with a double-Morse potential [31] can be extended to the $2 \mathrm{C}$ topological (anti)kinks. More precisely, it should be examined how the softness of the background sublattice affects the bifurcation structure and symmetry switchings of the proton (anti)kink states.

Another important specific consequence that arises owing to the softness of the background of a HB chain is the obvious difference of the kink and antikink profiles. This "kinkantikink asymmetry" originates from the dependence of the barrier height in the double-well proton potential on the distance between the nearest-neighbor ions $X^{-}$that form this potential and the self-consistent description of the proton-ion interaction. Indeed, under forming the $2 \mathrm{C}$ kink state, the proton sublattice is locally stretched at the kink center implying in this region a localized contraction of the heavy-ion sublattice. As a result, the barrier for proton transfers that depends on the distance between the adjacent heavy ions increases for the kink. This is an intuitive argument and therefore the opposite situation seems to occur in the central region of the $2 \mathrm{C}$ antikink. In fact, as shown below in this paper by using numerically exact methods, the $2 \mathrm{C}$ antikink profiles in both the "optical" $2 \mathrm{CO}$ and "acoustic" $2 \mathrm{CA}$ chains appear to be rather sophisticated with unexpected symmetries and topologies. This is because of a nontrivial balance of forces in the $2 \mathrm{C}$ chains. As regards the (anti)kink mobility, it is expected (see also Ref. [26]) that the mobility of the $2 \mathrm{C}$ kink (negative ionic defect) should be lower than that of the antikink (positive ionic defect). Indeed, such a situation may occur in realistic systems. Thus, as known from experiments [56], the mobility of the positive ionic defects in ice exceeds by one order the mobility of the negative ionic defects. This phenomenon of asymmetric behavior of the $2 \mathrm{C}$ antikinks (positive defects) and the 2C kinks (negative defects) has been confirmed by molecular dynamics simulations previously [26] using a general $2 \mathrm{C}$ model for proton transport being a combination of the $2 \mathrm{CO}$ and $2 \mathrm{CA}$ models with additional nonlinearities involved.

The paper is organized as follows. In the next section, we present the equations of motion for a general $2 \mathrm{C}$ chain model that comprises both the $2 \mathrm{CO}$ and 2CA models and discuss the spectrum of small-amplitude oscillations. In Sec. III, we find the discrete profiles of stationary kink and antikink solutions, and analyze stability switchings for the $2 \mathrm{CO}$ and 2CA chains. Possible applications of the stability switching effect are discussed in Sec. IV. Conclusions are given in Sec. V.

\section{THE TWO-COMPONENT MODEL}

The $2 \mathrm{C}$ chain model that describes the dynamics of the proton and ion interacting sublattices is a one-dimensional diatomic chain of coupled particles with two alternating masses, $m_{p}$ and $M$ being the masses of the proton and the heavy ion, respectively. The ion and proton subsystems interact with each other via the intrabond proton potential formed by the neighboring ions. The assumption of fixed ions can formally be reached in the limit $M \rightarrow \infty$, resulting in the 1C (monoatomic) model, wherein only the proton part of the Hamiltonian [31] has been taken into consideration. The total two-sublattice Hamiltonian that includes both the $2 \mathrm{CO}$ and 2CA models can be written in the form

$$
\begin{aligned}
H= & \sum_{n}\left[\frac{1}{2} \dot{q}_{n}^{2}+\frac{\mu}{2} \dot{Q}_{n}^{2}+\frac{\kappa_{p}}{2}\left(q_{n+1}-q_{n}\right)^{2}+\frac{\kappa_{i}}{2}\left(Q_{n+1}-Q_{n}\right)^{2}\right. \\
& \left.+\frac{\kappa_{\mathrm{o}}}{2} Q_{n}^{2}+V\left(u_{n}, \rho_{n}\right)\right],
\end{aligned}
$$

where the heavy ions and protons are labeled according to the sequence

$$
\left\{\ldots ; Q_{n-1}, q_{n-1} ; Q_{n}, q_{n} ; Q_{n+1}, q_{n+1} ; \ldots\right\} .
$$

Here and in what follows we adopt the dimensionless description, where $q_{n}$ is a dimensionless proton displacement of the $n$th proton in the hydrogen bridge from the middle of the $n$th and $(n+1)$ th heavy ions, when these ions are in equilibrium positions; $Q_{n}$ is a dimensionless displacement of the $n$th heavy ion from its equilibrium position; and $\mu=M / m_{p}$ the relative heavy-ion mass. The displacements $Q_{n}^{\prime} \mathrm{s}$ and $q_{n}^{\prime}$ s are scaled by the lattice constant $l$. The overdot denotes the differentiation with respect to the dimensionless time $\tau=t / t_{0}$, where $t_{0}=l \sqrt{m_{p} / \varepsilon_{0}}$, with $\varepsilon_{0}$ being the activation energy for proton transfers over the barrier in the HB bridge when all the heavy ions are fixed at a distance (lattice spacing) $l$. The coupling constants are given in the dimensionless form $\kappa_{p}$ $=K_{p} l^{2} / \varepsilon_{0}$ (coupling between the protons in the adjacent $\mathrm{HB}$ bridges), $\kappa_{i}=K_{i} l^{2} / \varepsilon_{0}$ (coupling between the adjacent ions), and $\kappa_{o}=K_{o} l^{2} / \varepsilon_{0}$ (coupling of the ions with the on-site potential). The two-dimensional intrabond proton potential $V(u, \rho)$ is assumed to be a symmetric double-well function (with respect to variable $u$ ) of a general form with two minima at $(u, \rho)=( \pm a, 0)$ (more details on the topology of this potential are given in Ref. [30]). The lattice variables $u_{n}$ and $\rho_{n}$ are defined through the relations

$$
\begin{gathered}
u_{n}=q_{n}-\frac{1}{2}\left(Q_{n}+Q_{n+1}\right), \\
\rho_{n}=Q_{n+1}-Q_{n} .
\end{gathered}
$$

Similarly to Ref. [31], in order to have the explicit expression for $V(u, \rho)$, we choose a pair of Morse potentials, placed tail-to-tail as shown in Fig. 1, resulting in

$$
V(u, \rho)=\left[\frac{\alpha-\cosh (\beta u) e^{-\beta \rho / 2}}{\alpha-1}\right]^{2},
$$

where the parameter $\beta$ measures the curvature along the $u$ direction of the potential surface at $\rho=0$. The parameter $\beta$ is adjustable, while the other parameter $\alpha$ can be computed through the equilibrium distance $r_{0}$ of a single Morse potential $[26,30]$ using the relation 


$$
\alpha=\frac{1}{2} \exp \left[\beta\left(\frac{1}{2}-r_{0}\right)\right] .
$$

Then the two equilibrium positions $\pm a$ (at $\rho=0$ ) are found from the equation $\alpha=\cosh (\beta a)$. Throughout the paper we take $r_{0}=0.25$ and $\mu=17$ (as an example of the hydroxide ion $\mathrm{OH}^{-}$). The equations of motion that correspond to the Hamiltonian (1) take the form

$$
\begin{aligned}
\ddot{q}_{n}= & \kappa_{p}\left(q_{n+1}-2 q_{n}+q_{n-1}\right)-\frac{\partial}{\partial q_{n}} V\left(u_{n}, \rho_{n}\right), \\
\ddot{Q}_{n}= & \left(\kappa_{i} / \mu\right)\left(Q_{n+1}-2 Q_{n}+Q_{n-1}\right)-\left(\kappa_{o} / \mu\right) Q_{n} \\
& -\frac{1}{\mu} \frac{\partial}{\partial Q_{n}}\left[V\left(u_{n-1}, \rho_{n-1}\right)+V\left(u_{n}, \rho_{n}\right)\right] .
\end{aligned}
$$

Before studying the 2C (anti)kink states, let us consider the spectrum of small-amplitude oscillations. After the linearization of the potential (3) around one of the global minima $(u, \rho)=( \pm a, 0)$ and inserting this linearized expression into Eqs. (5) and (6), we get the following dispersion law:

$$
\omega_{ \pm}^{2}(k)=\mathcal{A}(k) \pm \sqrt{\mathcal{A}^{2}(k)-2 \mathcal{B}(k)},
$$

where

$$
\begin{gathered}
\mathcal{A}=\left(\kappa_{p}+\kappa_{i} / \mu\right)(1-\cos k)-C(1+\cos k) / 2 \\
+C\left[\alpha^{2}+\left(\alpha^{2}-1\right) \mu\right]+\kappa_{o} / 2 \mu \\
\mathcal{B}=(1-\cos k)\left[2(1-\cos k) \kappa_{i}+\kappa_{o}\right] \kappa_{p} / \mu+C\left(\alpha^{2}-1\right) \\
\times\left[2(1-\cos k) \kappa_{i}+\kappa_{o}\right]+C\left(2 \alpha^{2}-1-\cos k\right) \\
\times(1-\cos k) \kappa_{p}, \\
C=\frac{\beta^{2}}{\left(\alpha^{2}-1\right) \mu} .
\end{gathered}
$$

Similarly to the standard case of the diatomic chain with only a nearest-neighbor coupling involved, the dispersion relation (7) also consists of two parts: the low-frequency branch $\omega_{-}(k)$ and the high-frequency branch $\omega_{+}(k)$. These branches are depicted in Fig. 3 for both the $2 \mathrm{CO}\left(\kappa_{i}=0\right.$ but $\left.\kappa_{o} \neq 0\right)$ [see panels (a) and (b)] and 2CA $\left(\kappa_{i} \neq 0\right.$ but $\left.\kappa_{o}=0\right)$ [see panels (c) and (d)]. In the former case, all the oscillations are of the optical type and therefore the $2 \mathrm{CO}$ chain is "closer" to the $1 \mathrm{C}$ model [31] than the 2CA chain.

As the system parameters vary, both the branches change their shape getting more flat or steep as demonstrated by Fig. 3(b), but due to "repulsion" between them, they do not intersect. In the latter case, when the chain is isolated $\left(\kappa_{o}=0\right)$, the upper branch describes the dispersion law of the optical small-amplitude oscillations while the lower branch is of the acoustic type (zero frequency at $k=0$ ). The $2 \mathrm{C}$ model given by the Hamiltonian (1) contains two characteristic velocities of small-amplitude waves in each of the sublattices: $c_{0}$ $\equiv l \sqrt{K_{p} / m_{p}}$ (in the proton sublattice) and $v_{0} \equiv l \sqrt{K_{i} / M}$ (in the ion sublattice). In the dimensionless description, these velocities are measured in units $l / t_{0}: s_{p} \equiv c_{0} t_{0} / l=\sqrt{\kappa_{p}}$ and $s_{i}$ $\equiv v_{0} t_{0} / l=\sqrt{\kappa_{i} / \mu}$. In general, the two cases $c_{0}>v_{0}$ and $c_{0}<v_{0}$
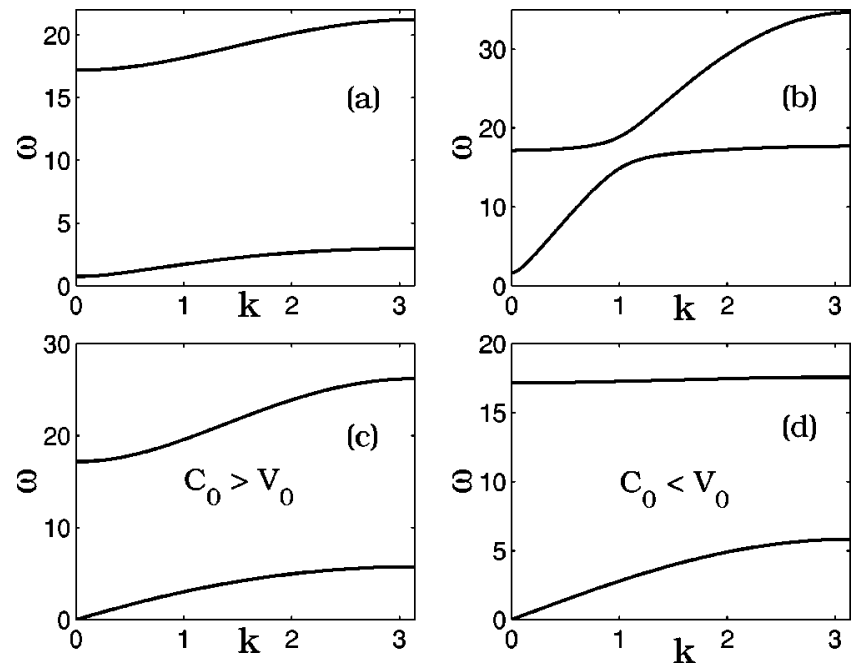

FIG. 3. Two bands of small-amplitude oscillations for $\beta=10$ : (a) $\kappa_{p}=10, \kappa_{i}=100$, and $\kappa_{o}=10$; (b) $\kappa_{p}=10, \kappa_{i}=5000$, and $\kappa_{o}=10$; (c) $\kappa_{p}=50, \kappa_{i}=100$, and $\kappa_{o}=0$; (d) $\kappa_{p}=3, \kappa_{i}=150$, and $\kappa_{o}=0$.

may be considered, depending on the ratio of $\kappa_{p}$ and $\kappa_{i}$. The spectra of small-amplitude oscillations in the 2CA chain are depicted in panels (c) and (d) of Fig. 3. For the acoustic branch in the 2CA chain, the phase velocity of the smallamplitude waves $\left[s_{-}=\omega_{-}(k) / k\right]$ attains a maximum in the limit $k \rightarrow 0$. Its explicit value is

$$
s_{\max }=\sqrt{\frac{\kappa_{p}+\kappa_{i}}{1+\mu}} .
$$

Note that the velocity (9) coincides with the lower edge of the second band of admissible kink velocities in the continuum limit [30].

\section{DISCRETE KINK STATES, THEIR STABILITY, AND BIFURCATIONS}

All the possible (stable and unstable) stationary profiles of the proton (anti)kink have been classified previously [31] on the basis of the $1 \mathrm{C}$ model. In particular, it has been shown that besides the (anti)kink states with a symmetric profile centered at a heavy ion or a proton, there exist two other types of stationary solutions: (i) (anti)kinks with an asymmetric profile and (ii) symmetric (anti)kinks with a zigzaglike profile. A close connection between the symmetric and asymmetric solutions has been shown to exist in the region, where the ion-centered and proton-centered (anti)kink solutions switch their stability. In the exactly solvable limit $\beta$ $\rightarrow \infty$, one can write down explicitly the expression for the (anti)kink profile (see also Ref. [57]). The (anti)kink profile consists of three distinct parts: the left and right tails, where $u_{n}$ attains exactly one of the two minima of the double-well potential, and the core, where an arbitrary finite number $(m$ $=0,1, \ldots)$ of protons can be found. The protons lie on the barrier of the double-well proton potential, whose value is constant in the interval $(-a, a)$ [see Eq. (3) as $\beta \rightarrow \infty$ ]. In this particular case, the stationary (anti)kink solutions can easily be found from the equations of motion (5), fixing the heavy- 
ion positions with $\rho_{n} \equiv 0$. Then the energy of the (anti)kink states in this limit can be calculated from the Hamiltonian (1) for any number of protons on the barrier $m$,

$$
E_{m}\left(\kappa_{p}\right)=m+2 \kappa_{p} a^{2} /(m+1), \quad m=0,1, \ldots .
$$

Despite Eq. (10) being obtained in the limit that describes a mathematical idealization of the proton-ion interaction, nevertheless, it is very instructive because it gives in very simple terms an insight into the phenomenon of stability switchings of the (anti)kink stationary states. First, it is worth to notice that Eqs. (10) admit many (in fact, a countable set) kink solutions, each corresponding to an integer $m$ at a fixed $\kappa_{p}$. Obviously, in the limit of small $\kappa_{p}$, the states with smaller number of protons on the barrier $m$ have smaller energy, so that the state with $m=0$ is the most energetically favorable one. Second, the energy dependencies of these solutions start to intersect each other as long as $\kappa_{p}$ increases (this can easily be observed if the corresponding curves are plotted; see, e.g., Fig. 4 of Ref. [31] for illustration). Therefore, the crossing points of the lines given by Eqs. (10) with two different numbers of protons on the barrier, $m_{1}$ and $m_{2}$, which take place at certain critical values of $\kappa_{p}$, can be determined from the equation $E_{m_{1}}\left(\kappa_{p}\right)=E_{m_{2}}\left(\kappa_{p}\right)$. The most interesting case is that when the lines, which correspond to an (anti)kink state with the lowest energy and the first state excited, intersect. What is happening in the vicinity of this point is a pitchfork bifurcation. An initially unstable symmetric (anti)kink solution (e.g., proton-centered) becomes stable, and two asymmetric unstable (anti)kink solutions appear. This situation exists in some small interval of the parameter $\kappa_{p}$, after which the asymmetric (anti)kinks disappear and the previously stable symmetric solution (in this case, an ion-centered one) becomes unstable. If we take $\beta$ to be finite but large, the above-mentioned situation will persist, at least, for the lowenergy (anti)kink solutions. Some of the "remnants" of the lines given by Eqs. (10) will cross each other (while interchanging stability via pitchfork bifurcations) several times as long as $\kappa_{p}$ increases, the higher-energy kink states may be turned into zigzag (anti)kinks or simply disappear. As long as $\beta$ decreases, less and less number of the solutions with many particles on the barrier survive, while only the proton- and ion-centered states interchange their stability. Note that in the limit $\beta \rightarrow 0$ the potential (3) coincides with the well known $\phi^{4}$ model. In the limit, when the parameter $\beta$ is too small, the switchings disappear and the only "survivors" from the exactly solvable limit are the two (anti)kinks with different (proton- and ion-centered) symmetries. For all $\kappa_{p}$, their energies are different, so no switching takes place. Therefore we emphasize once more that the switchings, although not present in the conventional models such as the $\phi^{4}$ and sineGordon ones, is a generic effect, which happens in a wide class of nonlinear Klein-Gordon models if the barrier of the on-site potential (in our case, the intrabond proton potential) is flat enough.

Now we focus on numerical studies of kinks and antikinks in the $2 \mathrm{C}$ model for finite values of the curvature $\beta$. We treat separately the $2 \mathrm{CO}$ and $2 \mathrm{CA}$ diatomic chains. Figure 4 demonstrates the symmetric stationary states for the 2CO model. Note that due to the difference between the
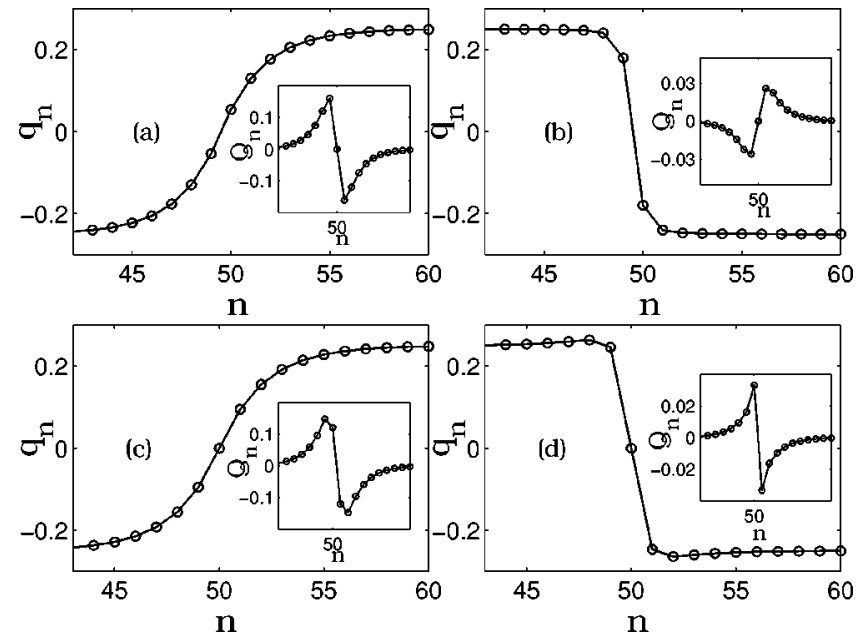

FIG. 4. Two-component profiles of monotonic symmetric (anti)kinks in the $2 \mathrm{CO}$ chain $\left(\kappa_{i}=0\right.$ and $\left.\kappa_{o}=5\right)$ with $\kappa_{p}=20$ and $\beta=5$ : (a) ion-centered kink, (b) ion-centered antikink, (c) proton-centered kink, and (d) proton-centered antikink.

profiles of the $2 \mathrm{C}$ kink and the $2 \mathrm{C}$ antikink, we study them separately.

In general, this difference, clearly demonstrated by Fig. 4 (we call it a "kink-antikink asymmetry"), follows from the asymmetric dependence of the double-Morse potential (3) on $\rho$ (at the point $\rho=0$ ). More precisely, due to the flatness of this potential, the effect of stretching the heavy-ion sublattice on increasing the barrier for proton transfers is not so crucial as a contraction of this sublattice on lowering this barrier. As a result, the $2 \mathrm{C}$ kinks in the $2 \mathrm{CO}$ model, where the boundaries are fixed, are rather wide, whereas the $2 \mathrm{C}$ antikinks are quite narrow (see Fig. 4). The insets show the profiles of the deformation of the heavy-ion component. Due to the fact that each ion is placed in the external on-site potential, the ion displacements tend asymptotically to zero (the ground state) as $n \rightarrow \pm \infty$. Note also the symmetry change of the ion component of the antikink profile, when its center being localized at an ion passes to a proton [compare panels (b) and (d) of Fig. 4]. Therefore the behavior of $2 \mathrm{C}$ (anti)kink profiles is not so obvious and simple as in 1C kink-bearing models.

The (anti)kink profiles for the 2CA chain are shown in Fig. 5. Here the difference between the kinks and antikinks is also present, but in another context. Owing to the absence of the on-site potential for the heavy ions, the localized deformation of the ion sublattice has a steplike profile, similarly to the proton component, but the orientation of these steps is again not obvious [compare panels (a) with (b) and (c) with (d) of Fig. 5 and note the same (monotonically decreasing) behavior of the ion-component profiles]. On the other hand, since the heavy-ion sublattice is isolated from any external forcing, the proton and ion displacements are arranged in such a self-consistent way that both the kinks and antikinks appear to be rather narrow, despite lowering the barrier height for proton transfers with decreasing the ion-ion distance. Intuitively, it seems that an opposite behavior should occur, but the total balance of forces in the $2 \mathrm{C}$ chain model appears to be rather complicated to draw correct qualitative conclusions. Notice also that for the heavy-ion sublattice in 

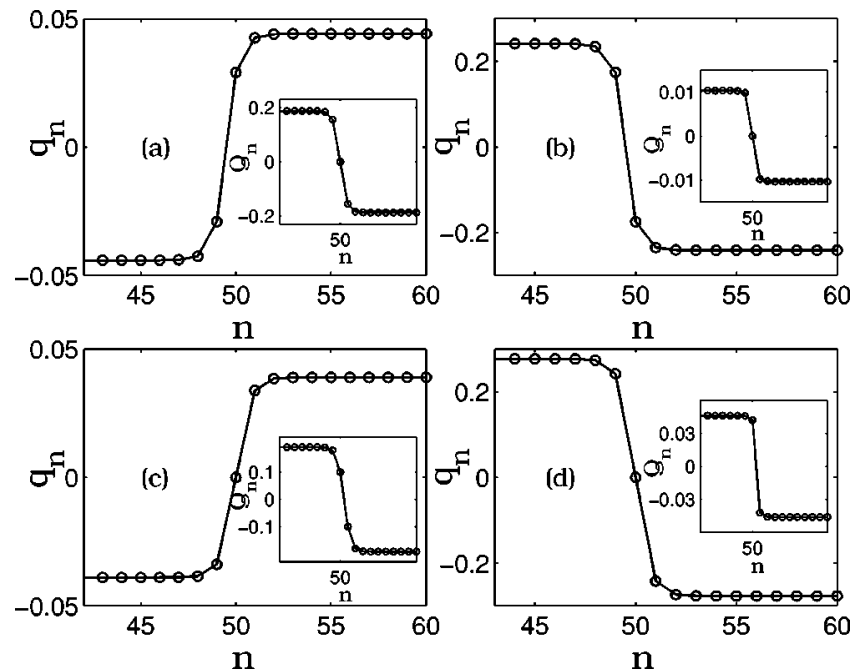

FIG. 5. Two-component profiles of monotonic symmetric (anti)kinks in the 2CA chain ( $\kappa_{i}=5$ and $\left.\kappa_{o}=0\right)$ with $\kappa_{p}=20$ and $\beta=5$ : (a) ion-centered kink, (b) ion-centered antikink, (c) proton-centered kink, and (d) proton-centered antikink.

the 2CO chain, the ion positions at the boundaries are fixed (like Dirichlet boundary conditions), whereas the boundary conditions for the ion sublattice in the 2CA chain are free (like Neumann boundary conditions).

\section{A. The $2 \mathrm{CO}$ chain model}

In this section, we deal with the stationary (anti)kink solutions for the $2 \mathrm{CO}$ chain $\left(\kappa_{i}=0\right.$ but $\left.\kappa_{o} \neq 0\right)$. Using the above-mentioned numerical techniques, we compute the kink and antikink solutions from the anticontinuous limit $\left(\kappa_{p}\right.$ $\rightarrow 0$ ). Varying the coupling constant $\kappa_{p}$ at a relatively weak interaction of the heavy ions with its substrate, $\kappa_{o}$, we check how the (anti)kink energies change. In Figs. 6 and 7, we plot the energy dependence on the coupling constant $\kappa_{p}$ at different curvatures $\beta$ for the antikink, and in Fig. 8 for the kink. These figures exhibit a close similarity to the results obtained for the 1C model [31]. Thus, in Fig. 6, curve 1 corresponds to the ion-centered antikink, whereas curve 2 corresponds to the proton-centered one. We see that before the point $\kappa_{p}$ $\simeq 41.55$, the energy of the ion-centered antikink is less than that of the proton-centered one, whereas the energies of these solutions coincide, when we pass this point. At higher values of $\kappa_{p}$, the situation becomes opposite; the energy of the proton-centered antikink is less than the energy of the ioncentered one. Therefore the interchanging of the antikink stability with the different symmetry after passing this point takes place.

For the 1C model [31], we have observed several switchings of stability, which take place along all the way up to the continuum limit. Unlike the $1 \mathrm{C}$ limit, in the $2 \mathrm{CO}$ chain, the number of switchings is determined by the interaction of the heavy-ion sublattice with the on-site potential. More precisely, when this interaction is sufficiently weak, we observe only one switching point. For stronger interaction, as we approach the 1C limit, the number of switchings increases. The region in the vicinity of the critical point is depicted in

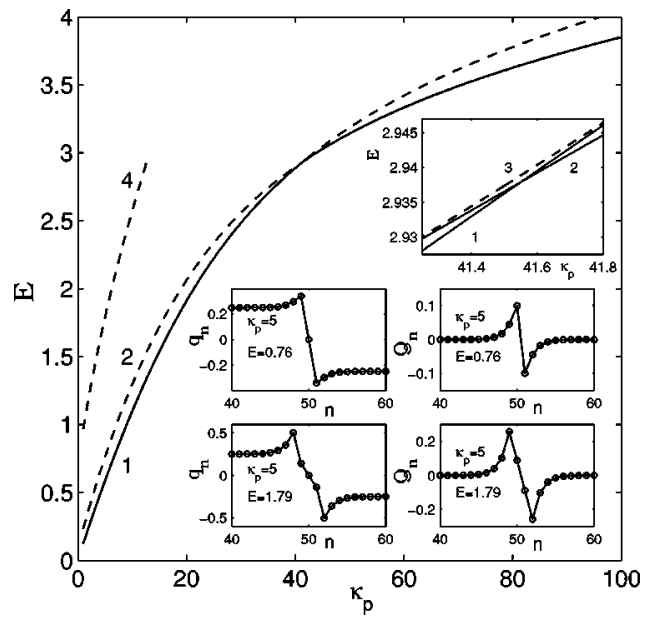

FIG. 6. Dependence of the energy of symmetric ion-centered antikink (curve 1) and symmetric proton-centered antikink (curves 2 and 4$)$ on coupling constant $\kappa_{p}$ in the $2 \mathrm{CO}$ chain $\left(\kappa_{i}=0\right.$ and $\kappa_{o}$ $=5$ ) with $\beta=5$. The upper inset shows more detailed behavior of the antikink energy $E$ in the vicinity of the stability switching, whereas curve 3 corresponds to the antikink with asymmetric profile. The lower four insets show the profiles of proton-centered antikinks at fixed $\kappa_{p}=5$. In all figures, solid lines correspond to stable and dashed lines to unstable states.

the upper inset of Fig. 6, where a new type of asymmetric stationary solutions represented by curve 3 is shown to connect the above-mentioned symmetric solutions via the bifurcation points. Therefore the bifurcation scenario in this case completely coincides with that found for the $1 \mathrm{C}$ model (see Fig. 8 of Ref. [31]).

Another (unstable) symmetric zigzaglike stationary antikink solution with higher energies is represented by curve 4 in Fig. 6. Note that for a sufficiently soft (e.g., $\kappa_{p} \sim 5$ ) $2 \mathrm{CO}$ chain, the proton component of the antikink solution takes a peculiar zigzaglike shape as illustrated in the two left lower insets of Fig. 6, contrary to the standard monotonically decreasing (antikink) shape known in the continuum limit. For stronger proton-proton couplings (e.g., $\left.\kappa_{p} \sim 20\right)$ the proton-

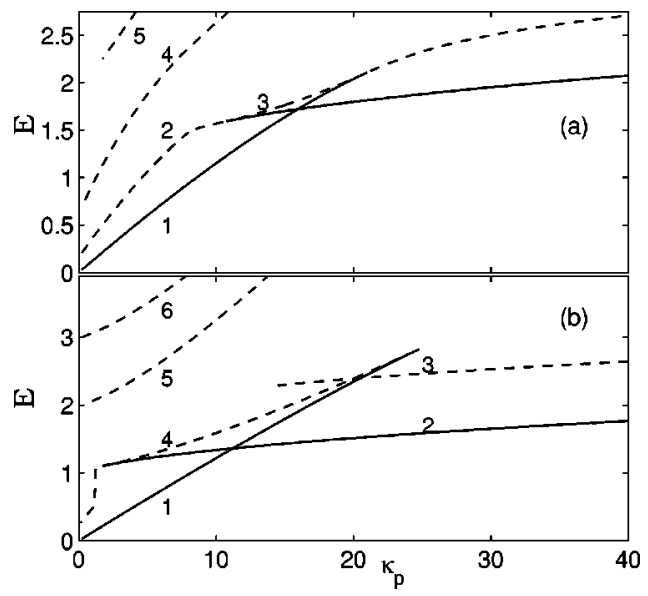

FIG. 7. Dependence of the antikink energy $E$ on the coupling constant $\kappa_{p}$ in the $2 \mathrm{CO}$ chain $\left(\kappa_{i}=0\right.$ and $\left.\kappa_{o}=5\right)$ with (a) $\beta=10$ and (b) $\beta=20$. Solid lines show stable and dashed lines unstable states. 


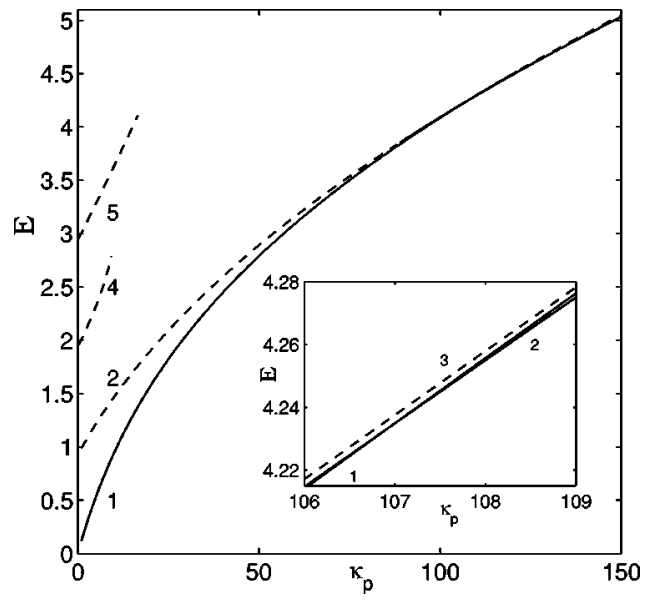

FIG. 8. Dependence of the kink energy $E$ on the coupling constant $\kappa_{p}$ in the $2 \mathrm{CO}$ chain $\left(\kappa_{i}=0\right.$ and $\left.\kappa_{o}=70\right)$ with $\beta=10$. Solid lines show stable and dashed lines unstable states.

component tails of the (proton-centered) antikink $2 \mathrm{CO}$ zigzaglike profile become more smooth as shown in Fig. 4(d). Thus, proton-centered antikinks appear to be deformed to this specific (zigzaglike) shape due to the deformation of the intrabond proton potential (3) caused by the ion displacements, although at stronger proton-proton couplings $\kappa_{p}$, the influence of the heavy-ion sublattice on the proton profiles practically vanishes.

In Fig. 7, we plot the dependence of the antikink energy on the coupling constant $\kappa_{p}$ for higher values of the curvature $\beta$. Here one can see that with the growth of $\beta$, the first switching occurs earlier being more pronounced. Curves 1 in panels (a) and (b) correspond to the ion-centered antikink and curves 2 to the proton-centered one. They cross each other at $\kappa_{p} \simeq 15.97$ and $\kappa_{p} \simeq 11.24$ for $\beta=10$ and $\beta=20$, respectively. One can see that for higher $\beta$, the bifurcation scenario remains the same, wherein the asymmetric antikink solutions represented by curve 3 in Fig. 7(a) and curve 4 in Fig. 7(b) play the same role.

In Fig. 7(b), one can observe the coexistence of the antikink solutions of the same symmetry with the different number of particles on the barrier. Curve 3 in this figure corresponds to the ion-centered antikink with two protons on the barrier. This coexistence originates from the limit $\beta \rightarrow \infty$. Zigzaglike antikinks also appear in these cases and they are represented by curves 4 and 5 in Fig. 7(a), and curves 5 and 6 in Fig. 7(b).

Figure 8 shows one switching for the kink stationary states. Here the transition of stability of the ion-centered kink (curve 1) and the proton-centered kink (curve 2) occurs at significantly stronger interaction of the ion sublattice with its substrate compared with the case of the antikink.

Thus, we have studied the dependence of the (anti)kink energy on the proton-proton coupling constant $\kappa_{p}$ in the $2 \mathrm{CO}$ model. As expected, similarly to the $1 \mathrm{C}$ chain for proton transfers, we have observed the stability (symmetry) switchings and the pitchfork bifurcations associated with them. However, in the case when the heavy-ion sublattice is soft (e.g., the constant $\kappa_{o}$ is not so large), this similarity takes place only for the antikink but not for the kink state. Only for

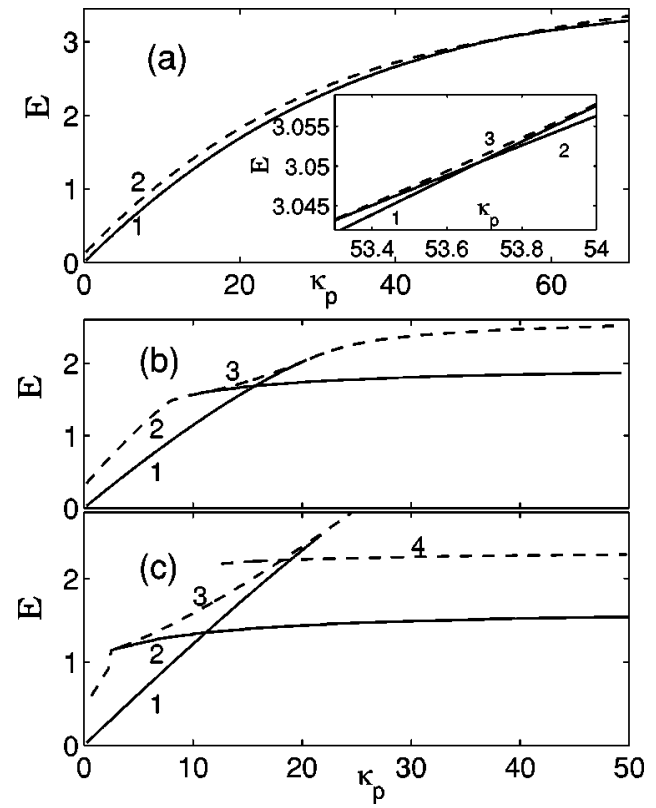

FIG. 9. Dependence of the antikink energy $E$ on the coupling constant $\kappa_{p}$ in the 2CA chain $\left(\kappa_{i}=5\right.$ and $\left.\kappa_{o}=0\right)$ with (a) $\beta=5$, (b) $\beta=10$, and (c) $\beta=20$. Solid lines show stable and dashed lines unstable states.

sufficiently large values of $\kappa_{o}$, the switchings also occur for the kink. In other words, in the limit $\kappa_{o} \rightarrow \infty$, the $2 \mathrm{CO}$ model is transformed into the $1 \mathrm{C}$ model, for which the switching effect has been studied in detail [31].

\section{B. The 2CA chain model}

In this section, we discuss the switching effect that takes place in the 2CA chain. Similarly to the $2 \mathrm{CO}$ chain, we have also investigated the same dependence of the (anti)kink energy on the proton-proton coupling $\kappa_{p}$ for different values of $\beta$. In Fig. 9, we show the antikink energy as a function of the proton-proton coupling constant $\kappa_{p}$. Panels (a), (b), and (c) correspond to the cases $\beta=5, \beta=10$, and $\beta=20$, respectively. In all the panels, curves 1 correspond to the ion-centered antikink, curves 2 to the proton-centered antikink, and curves 3 to the asymmetric antikink stationary solutions. For $\beta=5$, the first switching occurs at $\kappa_{p} \simeq 53.65$. At this critical point, the energies of both types of the antikink states coincide and after passing this point, the interchange of the stability of the ion-centered and proton-centered antikink states takes place. Here the bifurcation scenario is similar to that reported previously [31] for the $1 \mathrm{C}$ chain. At higher values of $\beta$, the first switching occurs earlier; for $\beta=10$, it happens at $\kappa_{p} \simeq 15$, and for $\beta=20$, it appears earlier. For such a large value of $\beta$, we have observed the coexistence of the states with the same symmetry, but with the different number of protons on the barrier; in Fig. 9(c), curve 4 corresponds to the ion-centered antikink solution with two protons on the barrier. However, this solution seems to be unstable for all values of $\kappa_{p}>0$.

Figure 10 illustrates one switching for the kink. One can see that the switching occurs at higher curvatures $\beta$, and the parameters of the interparticle interactions $\kappa_{p}$ and $\kappa_{i}$. Note 


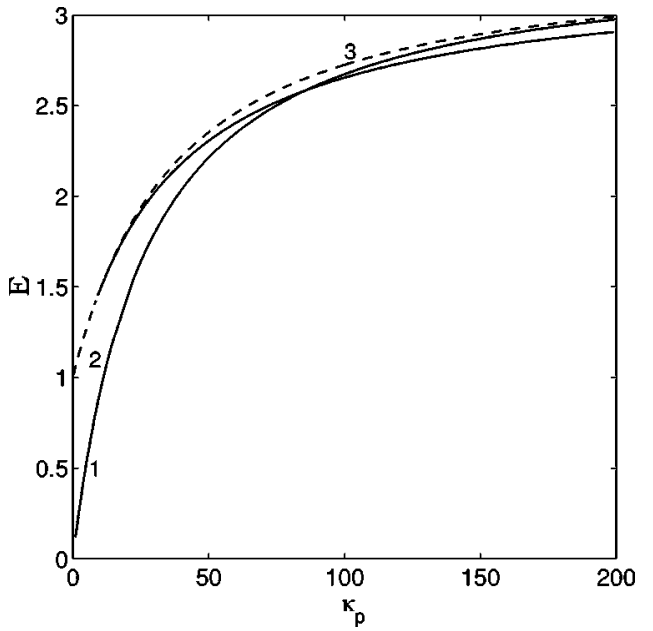

FIG. 10. Dependence of the kink energy $E$ on the coupling constant $\kappa_{p}$ in the 2CA chain $\left(\kappa_{i}=60\right.$ and $\left.\kappa_{0}=0\right)$ with $\beta=20$. Solid lines show stable and dashed lines unstable states.

that even at these large values, there are no intersection points between the ion-centered solution (curve 2) and the asymmetric solution (curve 3 ).

To summarize, we have seen that for the 2CA chain, some properties, which are characteristic for the $2 \mathrm{CO}$ chain, survive (such as stability switchings or the coexistence of two solutions of the same symmetry).

\section{PROTON TRANSPORT AND THE STABILITY SWITCHING EFFECT IN WATER-FILLED CARBON NANOTUBES}

The most interesting topic, where the discrete (anti)kink states may play an essential role, is proton conduction in $\mathrm{HB}$ chains of water molecules. Even though the (two-stage) mechanism of proton conduction in these chains was formulated long ago [1-5] that involves the propagation of two types of defects (ionic and bonding), the molecular basis for the fast proton translocation along HB chains is not yet fully understood. In the present paper, we restrict ourselves only to the study of the existence and stability properties of the ionic defects, which due to the cooperative nature of hydrogen bonding appear to be extended objects. The dynamics of these defects can be described in terms of the soliton theory, so that the extended positive and negative defects are nothing more than topological antikinks and kinks, respectively. More precisely, the (hydronium) $\mathrm{H}_{3} \mathrm{O}^{+}$ion can be treated as an ion-centered antikink, the (hydroxide) $\mathrm{OH}^{-}$ion an ioncentered kink, the Zundel ion $\left(\mathrm{H}_{5} \mathrm{O}_{2}^{+}\right)$a proton-centered antikink, and so on. Furthermore, one may also say that the so-called Grotthuss mechanism occurs only locally, in the region where the (anti)kink states are formed and stabilized.

For two decades, a linear HB chain of water molecules ("proton wire") was considered as a theoretical model for proton transfers across biological membranes [1], particularly through bacteriorhodopsin or half channels in the transmembrane $F_{0}$ portion of ATP synthase. However, exact data for interatomic forces are uncertain, except for recent $a b i n i$ tio results obtained by Pomès and Roux [12]. Besides the biological systems consisting of HB chains of water molecules, where discrete topological soliton solutions may play an essential role, at present carbon nanotubes filled by liquid water are of great interest $[13,14,16]$. These nanotubes show great potential for use in nanodevices. Nanotubes provide cylindrical channels similar to pipes used in the macroscopic world. Typically, the inner diameter of a carbon nanotube is less than $10 \mathrm{~nm}$. As a consequence, due to so small cross section, the interaction of water molecules with the nanotube walls is conceivably strong that prevents fluidic throughflow. This viewpoint has been supported by experiments on the behavior of water in nanosize channels [13]. In particular, it was shown that for extremely thin channels, which are comparable in size with interatomic distances, the continuum approach of fluid dynamics is not valid anymore. Moreover, the strong interaction between the water penetrated inside the tube and the wall appears to be of chemical nature. As a result, one-dimensional water wires are believed to be formed and the kink mechanism of proton transfers should take place in these systems. Recently $[14,16]$, this experimental evidence has been supported by $a b$ initio simulations of the dynamics of a carbon nanotube placed in a water reservoir. As observed from these studies, the initially empty central channel of the tube was rapidly filled by water from the reservoir, forming a preferentially aligned water wire with each water dipole oriented in the same direction parallel to the nanotube axis [see, e.g., Fig. 1(c) of Ref. [14]]. Carbon-water interface energies have been calculated, supporting a strong binding of an individual water molecule inside the nanotube to its wall. These studies have also shown the existence of stable complexes $\mathrm{H}_{2 n+1} \mathrm{O}_{n}^{+}$and single ions $\mathrm{OH}^{-}$.

As follows from the above description of structure and dynamics of water-filled carbon nanotubes, these systems can be modeled by the $2 \mathrm{CO}$ chain model. However, so far our study of both the $2 \mathrm{C}$ models has been performed in the dimensionless description that involves four parameters: the three stiffness constants of the ion-ion, ion-substrate, and proton-proton couplings $\kappa_{i, o, p}$ and the curvature of the Morse potential $\beta$. The study has been focused on the existence of switchings of stability, which occur at certain (critical) values of these parameters. In order to know whether these (dimensionless) values have any physical sense, we need to pass to the corresponding system parameters given in full dimensions, using the relations: $K_{i, o, p}=l^{-2} \varepsilon_{0} \kappa_{i, o, p}$ and $b$ $=\beta / l$ with $l$ being the length of the $X \cdots X$ bond and $\varepsilon_{0}$ the barrier height in the proton potential. Thus, according to the estimates based upon crystallographic and spectroscopic measurements for ice (see Ref. [26] and references therein), $l=2.76 \AA$ (the lattice constant for ice [56]), $\varepsilon_{0}=0.3 \mathrm{eV}, K_{p}$ $=22 \mathrm{~N} / \mathrm{m}, K_{i}=13 \mathrm{~N} / \mathrm{m}, K_{o}=4 \mathrm{~N} / \mathrm{m}$, and $b=7.4 \AA^{-1}$. This case corresponds to the following dimensionless values: $\kappa_{p}$ $=33.8, \kappa_{i}=20.0, \kappa_{o}=6.2$, and $\beta=20.4$. Some $a b$ initio calculations [27] give $b \simeq 2.7 \AA^{-1}$ for water systems, while $\varepsilon_{0}$ $\simeq 0.3 \mathrm{eV}$. Other computations [38] have determined the proton-proton interaction $K_{p} \simeq 80 \AA^{-2} \mathrm{kcal} / \mathrm{mol}$ $=56 \mathrm{~N} / \mathrm{m}\left(\kappa_{p} \simeq 60\right), \varepsilon_{0} \simeq 10 \mathrm{kcal} / \mathrm{mol}=0.4 \mathrm{eV}$.

On the other hand, the critical values at which the first switching of stability occurs in both the $2 \mathrm{C}$ chains can briefly 
be rewritten as follows. In the $2 \mathrm{CO}$ chain $\left(\kappa_{i}=0\right.$ and $\left.\kappa_{o}=5\right)$, for the antikink $\kappa_{p} \simeq 16$ if $\beta=10$ and $\kappa_{p} \simeq 11$ if $\beta=20$, whereas for the kink we have $\kappa_{o}=70$ and $\kappa_{p} \sim 100$ if $\beta=10$. In the 2CA chain $\left(\kappa_{o}=0\right.$ and $\left.\kappa_{i}=5\right)$, for the antikink $\kappa_{p}$ $\simeq 54,16$, and 11 if $\beta=5,10$, and 20 , respectively, whereas for the kink we have $\kappa_{i}=60$ and $\kappa_{p} \simeq 90$ if $\beta=20$. From the comparison of these values with the realistic (dimensionless) estimates given above $\left(\kappa_{o} \sim 6, \kappa_{i} \sim 20, \kappa_{p} \sim 30-60\right.$, and $\beta$ $\sim 7-20$ ) and the main result of Sec. III (according to which for larger $\kappa_{i, o}$ and $\beta$ the first switching of stability occurs at smaller $\kappa_{p}$ ), one can conclude that in realistic systems at least one switching may occur for the antikink, while for the kink such a switching seems to be hardly realized. Indeed, as illustrated by curves 1 and 2 of Figs. 6 and 8, for $\kappa_{p}$ $\sim 50-100$, with $\kappa_{o}=5$ and $\beta=10$, the proton-centered antikink that describes the $\mathrm{H}_{5} \mathrm{O}_{2}^{+}$ion and the ion-centered kink that corresponds to the $\mathrm{OH}^{-}$ion are stable. Moreover, the antikink mobility close to the point of stability switching is maximal, while the Peierls-Nabarro (PN) barrier for the $\mathrm{OH}^{-}$ ion is finite. This is why the molecular dynamics simulations by Savin and one of the authors (A.V.Z.) of the present paper [26] have revealed the higher (by one order) mobility of the positive ionic defects compared to the negative defects.

Finally, it should be noticed that the studies in this paper have been performed for wide regions of the system parameters, whereas in nature, particularly in biology, these parameters are fixed. These parameters might be varied under synthesis of nanofluidic devices, e.g., varying the occupancy of water molecules inside nanotubes and possibly the period $l$ (and therefore the parameter $\beta$ ) of a HB chain.

\section{CONCLUSIONS}

In this paper, we have studied the solitary wave excitations ( $2 \mathrm{C}$ kinks and antikinks), the whole variety of their narrow stationary profiles (states), switchings of their stable states to unstable ones and vice versa, and their propagation along a diatomic (2C) chain comprising the proton and heavy-ion sublattices. The heavy-ion component creates the two-dimensional potential $V(u, \rho)$, with proton $(u)$ and ion $(\rho)$ displacements, that plays a key role for proton transfers within the hydrogen bond. We have considered the function $V(u, \rho)$ as a $2 \mathrm{C}$ generalization of the intrabond proton potential constructed earlier [31] from two single Morse-type functions placed tail-to-tail at some distance, providing a double-well shape of the function $V(u, \rho)$ in the variable $u$ at $\rho=0$. As emphasized previously [30,31], this potential has been found to offer the best combination of accuracy in reproducing quantum-mechanically computed potentials $[27,29]$. The frequency spectrum and phase velocities of small-amplitude oscillations have also been studied for these models.

The studies here have mainly been done for two diatomic models of archetypal simplicity that admit $2 \mathrm{C}$ topological soliton solutions. The first of these is a diatomic chain, where each heavy ion is subject to an on-site potential of singleminimum topology, so that its linear two-band spectrum consists of only optical linear oscillations. The distance between the external sites provides the existence of the intrabond proton potential of a double-well form. The second one is the two-sublattice chain isolated from any external forces and therefore its lower branch of the linear spectrum is of acoustic type. These two chains have been referred above as to the $2 \mathrm{CO}$ and 2CA models, respectively.

Drawing the analogy between the $1 \mathrm{C}$ and $2 \mathrm{C}$ models, we have carried out a numerical study of stationary (anti)kink states in both the $2 \mathrm{CO}$ and $2 \mathrm{CA}$ chains. As a result, we have found that the stability switching effect also occurs in the two-sublattice chain. In this case, the number of switchings is determined by elasticity of the heavy-ion component; the stronger is the coupling of heavy ions either between them or with an external on-site potential, the larger is the number of stability switchings. This is the main result of our findings in the present paper because it was not possible at all to draw intuitively any conclusion on the behavior of the transparency regime (points or windows) on the elasticity properties of the background heavy-ion sublattice. Moreover, one could think that due to the softness of the background heavy-ion chain, even windows of finite size might exist in the (anti)kink transparency along the $2 \mathrm{C}$ chain used to describe the proton transport in HB chains.

Similarly to the $1 \mathrm{C}$ model, in the $2 \mathrm{C}$ chain, the asymmetric and zigzaglike $2 \mathrm{C}$ (anti)kink solutions have been shown to exist. The bifurcation scenario of stability switchings in the $1 \mathrm{C}$ and $2 \mathrm{C}$ models is the same and therefore we did not focus on this issue in the present paper. However, in the $2 \mathrm{C}$ chain, we were dealing with a kink-antikink asymmetry, the property being absent in the $1 \mathrm{C}$ chain. As a result of this asymmetry, the stability switchings for the kink and the antikink occur at different values of the system parameters. Since the PN barrier at the stability switching points practically vanishes, on the basis of the results of Sec. III one can conclude that the mobility of the kink is always lower than the mobility of the antikink in both the $2 \mathrm{CO}$ and $2 \mathrm{CA}$ chains. This result supports the molecular dynamics simulations for the $2 \mathrm{C}$ model, where the proton-proton coupling was of the Coulomb type [26]. The main feature of the $2 \mathrm{C}$ model lies in lowering the intrabond barrier in the hydrogen bond by virtue of the heavy ions. As a result of this barrier change, one can observe the lowering of the energy states and deviations of the ion- and proton-centered (anti)kink profiles from the corresponding $1 \mathrm{C}$ configurations. Owing to softness of the diatomic chain, the $2 \mathrm{C}$ profiles appear to be rather narrow. Moreover, their shape appears to be quite sophisticated and not so obvious and "monotonic" as in the corresponding $1 \mathrm{C}$ kink-bearing models, crucially depending on the model parameters [notice, for instance, the "anomalous" symmetry of the $Q_{n}$ profiles plotted in Figs. 4(d), 6(b), and 6(d) as well as the $q_{n}$ profiles shown in the insets of Fig. 7].

Finally, one should emphasize that the role of onedimensional chains of $\mathrm{HB}$ water molecules in mediating proton transfers through membranes is of fundamental importance not only in a number of biological systems, but also in nanotechnological processes. Since the discovery of carbon nanotubes, which can serve as models for biological proton channels, many interesting properties of these tubes have been revealed. In particular, recent experiments and simulations [12-16] showed that water can fill nanotubes forming 
HB chains that provide excellent conductors for proton currents through pores across membranes. Protons are believed to be conducted along the chain of water molecules inside a pore without the movement of the heavy (oxygen) atoms or ions, according to the soliton mechanism. The heavy ions only displace from their equilibrium positions, changing the shape of the double-well potential for proton transfers in the hydrogen bridges. At those critical parameter values, at which the symmetry switching of stability of (anti)kink states occurs and the PN relief disappears, the proton conduction becomes anomalous. This interesting property rigor- ously studied in the present paper from a general point of view may be used in making various nanofluidic devices.

\section{ACKNOWLEDGMENTS}

This work has benefited from stimulating and valuable discussions with M. Peyrard. The authors acknowledge partial support from the European Union under INTAS Grant No. 97-0368 and the RTN Project LOCNET HPRN-CT1999-00163, and the Danish Natural Science Research Council Grant No. 21-02-0500.
[1] J. F. Nagle and S. Tristram-Nagle, J. Membr. Biol. 74, 1 (1983).

[2] J. F. Nagle and H. J. Morowitz, Proc. Natl. Acad. Sci. U.S.A. 75, 298 (1978).

[3] J. F. Nagle, M. Mille, and H. J. Morowitz, J. Chem. Phys. 72, 3959 (1980).

[4] K. D. Kreuer, Chem. Mater. 8, 610 (1996).

[5] G. Zundel, Adv. Chem. Phys. 111, 1 (2000).

[6] H. Terao, T. Sugawara, Y. Kita, N. Sato, E. Kaho, and S. Takeda, J. Am. Chem. Soc. 123, 10468 (2001).

[7] L. Baroni, A. Cuccoli, V. Tognetti, and R. Vaia, J. Phys.: Condens. Matter 7, L625 (1995)

[8] P. M. Tomchuk and V. V. Krasnoholovets, J. Mol. Struct. 416, 161 (1997).

[9] P. M. Tomchuk and S. P. Luk'yanets, J. Mol. Struct. 513, 35 (1999); Phys. Status Solidi B 218, 291 (2000).

[10] N. I. Pavlenko, Phys. Rev. B 61, 4988 (2000); Phys. Status Solidi B 218, 295 (2000); J. Phys.: Condens. Matter 15, 291 (2003).

[11] F. Fillaux, J. Mol. Struct. 615, 45 (2002).

[12] R. Pomés and B. Roux, Biophys. J. 75, 33 (1998); 82, 2304 (2002).

[13] Y. Gogotsi, J. A. Libera, A. Güvenç-Yazicioglu, and C. M. Megaridis, Appl. Phys. Lett. 79, 1021 (2001).

[14] G. Hummer, J. C. Rasaiah, and J. P. Noworyta, Nature (London) 414, 188 (2001).

[15] C. Dellago, M. M. Naor, and G. Hummer, Phys. Rev. Lett. 90, 105902 (2003).

[16] D. J. Mann and M. D. Halls, Phys. Rev. Lett. 90, 195503 (2003).

[17] F. Zhu and K. Schulten, Biophys. J. 85, 236 (2003).

[18] N. Bjerrum, K. Dan. Vidensk. Selsk. Mat. Fys. Medd. 27, 1 (1951); Science 115, 385 (1952).

[19] J. H. Weiner and A. Askar, Nature (London) 226, 842 (1970).

[20] Y. Kashimori, T. Kikuchi, and K. Nishimoto, J. Chem. Phys. 77, 1904 (1982).

[21] V. Y. Antonchenko, A. S. Davydov, and A. V. Zolotaryuk, Phys. Status Solidi B 115, 631 (1983).

[22] S. Yomosa, J. Phys. Soc. Jpn. 52, 1866 (1983).

[23] A. V. Zolotaryuk and A. V. Savin, in Solitons and Applications, edited by V. G. Makhankov, V. K. Fedyanin, and O. K. Pashaev (World Scientific, Singapore, 1990), pp. 429-433.

[24] E. S. Kryachko, Chem. Phys. 143, 359 (1990).

[25] A. V. Zolotaryuk, St. Pnevmatikos, and A. V. Savin, Physica D
51, 407 (1991).

[26] A. V. Savin and A. V. Zolotaryuk, Phys. Rev. A 44, 8167 (1991).

[27] X. Duan and S. Scheiner, J. Mol. Struct. 270, 173 (1992).

[28] R. Grauer, K. H. Spatschek, and A. V. Zolotaryuk, Phys. Rev. E 47, 236 (1993).

[29] S. Scheiner and X. Duan, ACS Symp. Ser. 569, 125 (1994).

[30] A. V. Zolotaryuk, M. Peyrard, and K. H. Spatschek, Phys. Rev. E 62, 5706 (2000).

[31] V. M. Karpan, Y. Zolotaryuk, P. L. Christiansen, and A. V. Zolotaryuk, Phys. Rev. E 66, 066603 (2002).

[32] A. V. Zolotaryuk, K. H. Spatschek, and E. W. Laedke, Phys. Lett. 101A, 517 (1984).

[33] E. W. Laedke, K. H. Spatschek, M. Wilkens, Jr., and A. V. Zolotaryuk, Phys. Rev. A 32, 1161 (1985).

[34] A. V. Zolotaryuk, Teor. Mat. Fiz. 68, 415 (1986) [Sov. Theor. Math. Phys. 68, 916 (1987)].

[35] M. Peyrard, St. Pnevmatikos, and N. Flytzanis, Phys. Rev. A 36, 903 (1987).

[36] D. Hochstrasser, H. Büttner, H. Desfontaines, and M. Peyrard, Phys. Rev. A 38, 5332 (1988).

[37] J. Halding and P. S. Lomdahl, Phys. Rev. A 37, 2608 (1988).

[38] A. Gordon, Solid State Commun. 68, 885 (1988); 69, 1113 (1989); 72, 223 (1989).

[39] G. P. Tsironis and St. Pnevmatikos, Phys. Rev. B 39, 7161 (1989).

[40] St. Pnevmatikos, G. P. Tsironis, and A. V. Zolotaryuk, J. Mol. Liq. 41, 85 (1989).

[41] Y. S. Kivshar, Phys. Rev. A 43, 3117 (1991).

[42] B. A. Ivanov and G. K. Oksyuk, Phys. Lett. A 170, 63 (1992).

[43] J. M. Khalack and M. J. Velgakis, J. Phys.: Condens. Matter 14, 4747 (2002).

[44] E. S. Nylund and G. P. Tsironis, Phys. Rev. Lett. 66, 1886 (1991).

[45] R. Mefougue, P. Woafo, and T. C. Kofane, Solid State Commun. 86, 393 (1993).

[46] O. Yanovitskii, G. Vlastou-Tsinganos, and N. Flytzanis, Phys. Rev. B 48, 12645 (1993).

[47] E. Nylund, K. Lindenberg, and G. Tsironis, J. Stat. Phys. 70, 163 (1993).

[48] P. T. Dinda and C. R. Willis, Physica D 70, 217 (1994).

[49] C. M. Ngabireng, P. Woafo, and T. C. Kofane, Solid State Commun. 89, 885 (1994).

[50] P. Woafo, R. Takontchoup, and A. S. Bokosah, J. Phys. Chem. 
Solids 56, 1277 (1995).

[51] T. Cretegny and M. Peyrard, Phys. Lett. A 210, 347 (1996).

[52] I. A. Howard and R. Mittal, Phys. Rev. B 57, 45 (1998).

[53] I. A. Howard, R. Sharma, and R. Mittal, Physica D 113, 212 (1998).

[54] M. Peyrard and M. Remoissenet, Phys. Rev. B 26, 2886
(1982).

[55] M. Remoissenet and M. Peyrard, J. Phys. C 14, L481 (1981). [56] P. V. Hobbs, Ice Physics (Clarendon, Oxford, 1974).

[57] A. V. Savin, Y. Zolotaryuk, and J. C. Eilbeck, Physica D 138, 267 (2000). 